\title{
THE DARK ENERGY EXPLAINED BY A SCHWARZSCHILDIAN COSMOLOGY - ET CETERA
}

\author{
Nota del m.e. ANGELO LOINGER $(*)$ e di TIZIANA MARSICO (**)
}

(Adunanza del 13 marzo 2013)

SuNTO. - Nella prima parte della Nota si sviluppa una cosmologia schwarzschildiana, che, in virtù della repulsione gravitazionale scoperta da Hilbert, rende ragione sia dello spostamento verso il rosso delle righe spettrali delle galassie sia dell'esistenza dell'Energia Oscura. Nella seconda parte si discutono talune conseguenze - ignorate dalla letteratura relativistica - della metrica di Friedmann. -

ABstRact. - In a First Part we give a Schwarzschildian generalization of the Newtonian, and Friedmannian, models of universe. By virtue of the Hilbertian gravitational repulsion, our formulation explains the red-shifts of the galaxies and the existence of a DARK ENERGY. In the Second Part we discuss two ignored consequences of Friedmann's metrics: $i$ ) the existence of a time coordinate such that there is no expansion of Friedmann's universes; ii ) a corollary of a theorem by Hönl (1949), which tells us that the physical meaning of the point-origin of the galaxy paths is very problematic.

Introduction. - 1. The concept of a Schwarzschildian cosmology. - 2. Some results of the Newtonian and Friedmannian models of universe. -3 . Discussion of the properties of the Schwarzschildian galaxy trajectories. We prove that there exist values of the galaxy velocities which explain (without the intervention of the Lambda-term) the

(*) Dipartimento di Fisica, Università degli Studi di Milano, Italy.

E-mail: angelo.loinger@mi.infn.it

(**) Liceo Classico "G. Berchet", Via della Commenda 26, 20122 Milano, Italy.

E-mail: martiz64@libero.it 
galactic red-shifts and the existence of a DARK ENERGY. -4 . The concepts of inertial systems and inertial time according to the operational formulation by L. Lange. $\mathbf{- 5}$. The time coordinate $t^{\prime}$ for which there is no expansion of Friedmann's universe - and some consequences of this fact. - 5bis. The Hubble's law as a first approximation of Friedmann's treatment. The geodesic null-lines of Friedmann's metrics. - 5ter. A rèsumè of our results about galaxy red-shifts and Dark Energy. - 6. A comparison of the expansion "mechanisms" in the Newtonian, Friedmannian, and Schwarzschildian cosmologies. - 7. As a consequence of a theorem by Hönl, Friedmann's models of universe lose their physical meaning if $R \rightarrow 0$. - Appendix: Schwarzschildian models with a cosmological Lambda-term. -

INTRODUCTION. - The equations of motion of the Newtonian models [1] and of Friedmann's models (with zero pressure) [2] are mathematically isomorphic, as it is known (see, e.g., [3]). However, while Friedmann's metrics explain the observational red-shifts of the galactic spectral lines, the Newtonian models give Doppler red-shifts that are in some accord with the observations only if we add to the Newtonian equations of motion the cosmological $\Lambda$-term (see, e.g., [4]).

In the First Part of the present paper we develop a Schwarzschildian generalization of the Newtonian - and Friedmannian - models. Our results explain (without the $\Lambda$-term) the red-shifts of the galaxies and the Dark Energy : indeed, there exist (by virtue of Hilbert's gravitational repulsion [5]) innumerable galaxy trajectories which go to infinite with increasing galaxy velocities.

In the Second Part we discuss two consequences of Friedmann's metrics, which have not been considered in the relativistic literature: $i$ ) the existence of a time coordinate $t^{\prime}$ for which there is no expansion; $i$ ) a remarkable corollary of a result by Hönl (1949), which tells us that it is very problematic to assign a physical meaning to the common pointorigin of the galaxy trajectories.

\section{FIRST PART}

1. - As in the models cited in [1] and [2], in the following treatment the galaxies are represented by the particles of a (continuous) "dust" with a mass density $\varrho=\varrho(t)$. We start from the consideration of Schwarzschild's manifold created by a finite matter distribution about 
a centre with radial symmetry. As it is well known, the metric external to this spherical distribution is given by the following $\mathrm{d} s^{2}[6]$ :

$$
\mathrm{d} s^{2}=\left(1-\frac{\alpha}{\mathcal{R}}\right) c^{2} \mathrm{~d} t^{2}-\left(1-\frac{\alpha}{\mathcal{R}}\right)^{-1} \mathrm{~d} \mathcal{R}^{2}-\mathcal{R}^{2}\left(\mathrm{~d} \vartheta^{2}+\sin ^{2} \vartheta \mathrm{d} \varphi^{2}\right),
$$

where: $\mathcal{R}=\mathcal{R}(r)$ is any regular function of $r$ such that the metric becomes Minkowskian at the spatial infinite; $\alpha \equiv 2 m \equiv 2\left(G / c^{2}\right) M$, if $M$ is the mass of the gravitating distribution.

Let us imagine an expanding "cosmic sphere" composed of the mentioned "dust" particles. Let the radial coordinate of the spherical-surface points at time $t$ be $\mathcal{R}_{S}(t) \equiv \mathcal{R}_{S}[r(t)]$ - and denote with $\mathcal{R}_{I}=\mathcal{R}_{I}(t) \leq$ $\mathcal{R}_{s}(t)$ the radial coordinate of the surface points of any concentric internal sphere $I$. Then, $V\left(\mathcal{R}_{1}\right) \varrho(t)=M\left(\mathcal{R}_{I}\right)=\alpha_{I} c^{2} /(2 G)$, if $V\left(\mathcal{R}_{1}\right)$ is the volume of sphere $I$. In the sequel, we shall omit the index I and write simply $\mathcal{R}, \alpha$.

The trajectories of the galaxy particles are (radial) geodesics, because the matter tensor of the dynamical system is $T^{j k}=\varrho u^{j} u^{k},(i, k=$ $0,1,2,3)$, with $u^{j}$ a radial four-velocity. These geodesics are identical to the radial geodesics of the test-particles of metric (1).

We have a relativistic generalization of the Newtonian formulation [1], which is also more general than Friedmann's treatment [2].

2. - In the Newtonian formalism the velocity of a particle is $v=$ $\mathrm{d} R / \mathrm{d} t$, if $R=R(t)$ is its radial coordinate. In a "parabolic" motion we have $v(R \rightarrow \infty)=0$; in a "hyperbolic" motion we have $v(R \rightarrow \infty)=$ a constant $\neq 0$; in an "elliptic" case the particle describes a finite trajectory, first in the positive and then in the negative direction.

Analogous results in Friedmann's formalism for $\mathrm{d} R / \mathrm{d} t$, where now $\xi / R^{2}(t)$ is the curvature of the space section $t=$ constant $(\xi=0 ;-1 ;+1)$.

3. - We adapt to our treatment Hilbert's formulae for the radial geodesics of the test-particles of Schwarzschild's metric [5].

In lieu of his specific coordinate $r$, we employ the general coordinate $\mathcal{R}(r)$, denote with $-|A|$ his constant $A(<0)$, and write $c$ for the light speed in lieu of 1 . Then, Hilbert's eq. (54) becomes:

$$
\dot{\mathcal{R}} \equiv\left(\frac{\mathrm{d} \mathcal{R}}{\mathrm{d}(c t)}\right)^{2}=\left(\frac{\mathcal{R}-\alpha}{\mathcal{R}}\right)^{2}\left[1-|A|\left(\frac{\mathcal{R}-\alpha}{\mathcal{R}}\right)\right],
$$

where $\mathcal{R}$ is the radial coordinate of a generic galaxy of the expanding sphere. If $|A|=1$, we have the analogue of a "parabolic" trajectory: $\dot{\mathcal{R}}(\mathcal{R} \rightarrow \infty)=0$. 
If $|A|<1$, we have the analogue of a "hyperbolic" trajectory: $\dot{\mathcal{R}}(\mathcal{R} \rightarrow \infty)$ $=(1-|A|)^{1 / 2}$, a constant. In the analogue of the "elliptic" case we have $|A|$ $>1$, and $\mathcal{R}$ cannot be greater than $|A| \alpha /(|A|-1)$; the particle "ascends" as far as $\dot{\mathcal{R}}$ becomes zero (i.e., when $\mathcal{R}=|A| \alpha /(|A|-1)$ ), then it reverts its motion and travels again over the "ascensional" trajectory.

The acceleration $\ddot{\mathcal{R}}$ as a function of $A$ can be computed by deriving eq. (2) with respect to $t: \mathrm{d}\left(\dot{\mathcal{R}}^{2}\right) / \mathrm{d}(c t)=2 \dot{\mathcal{R}} \ddot{\mathcal{R}}$; we obtain:

$\ddot{\mathcal{R}}=\left(1-\frac{\alpha}{\mathcal{R}}\right) \alpha \mathcal{R}^{-2}-\frac{3|A|}{2}\left(1-\frac{\alpha}{\mathcal{R}}\right)^{2} \alpha \mathcal{R}^{-2} ;$

if $\ddot{\mathcal{R}}\left(\mathcal{R}_{*}\right)=0$, we have

$|A|=\frac{2}{3} \frac{\mathcal{R}_{*}}{\mathcal{R}_{*}-\alpha} \quad ; \quad \mathcal{R}_{*} \equiv \frac{3|A| \alpha}{3|A|-2}<\overline{\mathcal{R}} \equiv \frac{|A| \alpha}{|A|-1}$.

When $\mathcal{R}>\mathcal{R}_{*}$ there is attraction: $\ddot{\mathcal{R}}<0$; when $\mathcal{R}<\mathcal{R}_{*}$ there is repulsion: $\ddot{\mathcal{R}}>0$.

If $0 \leq|A| \leq 1$ no superior limit exists for the values of $\mathcal{R}$, and $\ddot{R}$ $(\mathcal{R} \rightarrow \infty)=0$.

The substitution of $\dot{\mathcal{R}}^{2}$ of eq. (2) in Hilbert's eq. (53):

$\ddot{\mathcal{R}}=\frac{3 \alpha}{2 \mathcal{R}(\mathcal{R}-\alpha)} \dot{\mathcal{R}}^{2}-\frac{\alpha(\mathcal{R}-\alpha)}{2 \mathcal{R}^{3}}$,

gives

$\ddot{\mathcal{R}}=\frac{3 \alpha}{2 \mathcal{R}(\mathcal{R}-\alpha)}\left\{\left(1-\frac{\alpha}{\mathcal{R}}\right)^{2}\left[1-|A|\left(1-\frac{\alpha}{\mathcal{R}}\right)\right]\right\}-\frac{\alpha(\mathcal{R}-\alpha)}{2 \mathcal{R}^{3}}$,

and we see again that $\mathcal{R} \leq|A| \alpha /(|A|-1)$ when $|A|>1$.

Eq. (5) tells us that we have attraction when

$|\dot{\mathcal{R}}|<\frac{1}{\sqrt{3}} \frac{\mathcal{R}-\alpha}{\mathcal{R}}$,

and repulsion when

$|\dot{\mathcal{R}}|>\frac{1}{\sqrt{3}} \frac{\mathcal{R}-\alpha}{\mathcal{R}}$.

Since $\ddot{\mathcal{R}}$ is zero when $\dot{\mathcal{R}}^{2}=(1 / 3)(\mathcal{R}-\alpha)^{2} / \mathcal{R}^{2}$, if we write

$\frac{1}{3}\left(\frac{\mathcal{R}-\alpha}{\mathcal{R}}\right)^{2}=\left(\frac{\mathcal{R}-\alpha}{\mathcal{R}}\right)^{2}\left[1-|A|\left(\frac{\mathcal{R}-\alpha}{\mathcal{R}}\right)\right]$,

we obtain obviously $\mathcal{R}=\mathcal{R}_{*} \equiv(3|A| \alpha) /(3|A|-2)$. 
If $0 \leq|A| \leq(2 / 3)$, the acceleration $\ddot{\mathcal{R}}$ is always different from zero and positive, there is repulsion for all the values of $\mathcal{R}$. The velocity $|\dot{\mathcal{R}}|$ is maximal at $\mathcal{R}=\infty$, where $|\dot{\mathcal{R}}(\infty)|=(1-|A|) 1 / 2$, i.e. $(1 / 3) \sqrt{3} \leq|\mathcal{R}(\infty)|$ $\leq 1$. (In particular, if $A=0$ we have $|\dot{\mathcal{R}}(\infty)|=1$.)

We see that there are values of the velocity which can explain the observational red-shifts of the galaxies by virtue of a Doppler effect. Of course, there are also galactic gravitational red-shifts, which are created by the mass $M$ of the expanding sphere.

Last but not least, we emphasize that our results give a clear explanation (without the cosmological $\Lambda$-term) of the observational existence of a Dark Energy [7].

At the end of the paper, the reader will find some diagrams of repulsion trajectories.

\section{SECOND PART}

4. - It is interesting to compare the concepts of spatial referenceframes and of time-scale in Newtonian and Friedmannian cosmologies.

For Newton's theory (and also for the special theory of relativity) the formulation of these concepts given by L. Lange in 1886 is the best [8]. It is composed of two definitions and two empirical propositions:

Definition I. - An inertial frame is every spatial coordinate system such that with reference to it the paths of three mass-points, projected from the same space point in three different directions, not belonging to the same plane, and then left to themselves, are straight lines. -

Empirical proposition J. - With reference to an inertial system, the path of every other mass-point left to itself is straight line. -

Definition II. - Inertial time-scale means every time-scale with respect to which a mass-point left to itself traverses equal distances in equal time intervals in its inertial path. -

Empirical proposition II. - With respect to an inertial time-scale, every other mass-point also traverses equal distances in its inertial path in equal time intervals. -

With Lange's formulation, Newton theory and the Newtonian cosmology do not need any metaphysical assumption, in particular the assumptions of an absolute space and an absolute time. The usefulness of the Copernican system rests on the experimental validity of the propositions $I$ and $I I$. 
5. - The fundamental formula of the Friedmannian red-shifts: $v(t) R(t)=$ constant, from which: $-\mathrm{d} v / v=\mathrm{d} R / R$, is usually demonstrated from the standpoint of geometrical optics, which is implicitly contained in the expression of the spatio-temporal interval $\mathrm{d} s$.

However, there is also a proof based on wave optics, which is particularly interesting from a physical point of view. It was obtained in 1931 by v. Laue [9]. He made a time transformation $t=t\left(t^{\prime}\right)$, where $t$ is Friedmann's time and $t^{\prime}$ is a suitable time such that with respect to it the homogeneous Maxwell equations assume a Minkowskian form. Thus, it is possible to write $F_{j k}^{\prime}=F_{j k}^{\prime(0)} \exp \left(i v^{\prime} t^{\prime}\right)$, where $F_{j k}^{\prime(0)}$ is the amplitude of the e.m. field $F_{j k}^{\prime}$ and $v^{\prime}$ is a constant frequency. Then, v. Laue comes back to Friedmann's time $t$, and remarking that $R(t)$ changes slowly with respect to the e.m. oscillations, he can conclude that $v(t)=$ $v^{\prime} c / R(t)$, from which $v(t) R(t)=$ constant; q.e.d. -

Now, it seems to us that the equations $F_{j k}^{\prime}=F_{j k}^{(0)} \exp \left(i v^{\prime} t^{\prime}\right)$ give an important conceptual information: there exists a time coordinate $t^{\prime}$ with respect to which we have no expansion of the universes. In reality, Friedmann's time coordinate $t$ is - with a good approximation - relative to an observer residing in a given, but whatever, galaxy (e.g., the Milky Way). (Remark that time $t$, by virtue of the Gaussian form of Friedmann's $\mathrm{d} s^{2}$ has a primordial character with respect to the proper times of the galaxies.) In conclusion, Friedmann's models of universe are, in a sense, "Ptolemaic", i.e. relative to the observational data recorded by galactic observers. And, of course, Friedmann's age of the universe has a value that is dependent on Friedmann's Gaussian frame, and therefore it is devoid of an invariant meaning.

5bis. - It was emphasized, in particular, by McVittie [10] that some commonly adopted quantities of Friedmann's formulation as: Hubble's parameter (or Hubble's "constant"), acceleration parameter, deceleration factor, etc., are approximate notions, that can be properly defined by a suitable series expansion of $1 / R(t)$.

If the indices $o$ and $s$ denote, resp., the observer and a light source, the fundamental formula of the cosmological red-shift: $v(t) R(t)$ $=$ constant tells us that

$\frac{\nu_{o}}{\nu_{s}}=\frac{R_{s}}{R_{o}}$,

from which 
$z=\frac{R_{o}}{R_{s}}-1$

Let us call: $t_{d}$ the departure-time instant of light; $\tau$, its travel-time; $t_{d}$, its arrival-time instant. We have: $R\left(t_{d}\right)=R\left(t_{o}-\tau\right) ; R\left(t_{o}\right) \equiv R_{o}$, and the following series $(\dot{R} \equiv \mathrm{d} R / \mathrm{d} t)$ :

$\frac{1}{R_{s}\left(t_{d}\right)}=\frac{1}{R_{o}}+\tau \frac{(\dot{R})_{o}}{R_{o}^{2}}+\frac{\tau^{2}}{2}\left[-\frac{(\ddot{R})_{o}}{R_{o}^{2}}+2 \frac{\left(\dot{R}^{2}\right)_{o}}{R_{o}^{3}}\right]+\ldots ;$

Hubbles's “constant”:

$h_{1}:=\frac{(\dot{R})_{o}}{R_{o}}$,

acceleration parameter:

$h_{2}:=\frac{(\ddot{R})_{o}}{R_{o}}$,

deceleration factor:

$q_{o}:=-\frac{(\ddot{R})_{o}}{R_{0}}\left[\frac{R_{o}}{(\dot{R})_{o}}\right]^{2} ;$

from which:

$$
z=h_{1} \tau+h_{1}^{2}\left(2+q_{o}\right) \frac{\tau^{2}}{2}+\ldots
$$

The first term gives the Hubble's law. -

Finally, we recall that the $\mathrm{d} \mathrm{s}^{2}$ of Friedmann's metrics can be written, with generic dimensionless space-coordinates $x^{1}, x^{2}, x^{3}$, in the following way:

$$
\begin{aligned}
& \mathrm{d} s^{2}=c^{2} \mathrm{~d} t^{2}-R^{2}(t) \mathrm{d} o^{2}, \\
& \mathrm{~d} o^{2}:=\gamma_{\alpha \beta}\left(x^{1}, x^{2}, x^{3}\right) \mathrm{d} x^{\alpha} \mathrm{d} x^{\beta} \quad, \quad(\alpha, \beta=1,2,3) .
\end{aligned}
$$

The equation $\mathrm{d} s^{2}=0$ of the null-geodesics tells us that

$$
\left|\frac{\mathrm{d} o}{\mathrm{~d} t}\right|=\frac{c}{R(t)}
$$

from which: 
$\left|\frac{\mathrm{d} o}{\mathrm{~d} t}\right|_{(R \rightarrow \infty)}=0 \quad ; \quad\left|\frac{\mathrm{d} o}{\mathrm{~d} t}\right|_{(R \rightarrow 0)}=\infty$.

5ter. - In our approach the accord with the observations concerning the cosmological red-shifts is certainly assured by the Doppler effect of the speeds on repulsive trajectories - and also by a gravitational Schwarzschildian effect, created by the total mass of the moving galaxies. However, the very novelty of our models resides in their plain explanation of the Dark Energy, explanation that follows from a concept of general relativity (Hilbertian repulsion), without the intervention of the cosmological $\Lambda$-term.

6. - In the Newtonian and Schwarzschildian cosmologies the expansion of the universe is generated by radial motions of the galaxies. In Friedmannian cosmology the galaxies have time-independent spatial coordinates, but the three-dimensional space is subjected to progressive dilatations (or contractions). The equations of motions of Newtonian and Friedmannian formulations are mathematically equivalent. Our formulation is more general, and contains them as approximations.

7. - We shall now expound a simple theorem by Hönl [11], that has an interesting consequence regarding the meaning of the origin, $R(t$ $=0)=0$, of Friedmann's expansion of the universe.

Let us write the $\mathrm{d} o^{2}$ of eq. (16) in angular coordinates $\vartheta, \varphi$, and $\Theta$ $(0 \leq \Theta \leq \pi)$ :

$$
\mathrm{d} o^{2}=\mathrm{d} \Theta^{2}+\left\{\begin{array}{l}
\sin ^{2} \Theta \\
\sinh ^{2} \Theta \\
\Theta^{2}
\end{array}\right\}\left(\mathrm{d} \vartheta^{2}+\sin ^{2} \vartheta \mathrm{d} \varphi^{2}\right),
$$

where $\sin ^{2} \Theta, \sinh ^{2} \Theta, \Theta^{2}$ correspond, respectively, to positive, negative, zero spatial curvature. The trajectories of the galaxies are geodesic lines characterized by $\Theta=$ const, $\vartheta=$ const, $\varphi=$ const.

Hönl considers a geodesic of a test-particle such that $\vartheta=$ const, $\varphi$ =const, $\Theta=\Theta(t)$. From

$$
0=\delta \int \frac{\mathrm{d} s}{\mathrm{~d} t} \mathrm{~d} t=\delta \int\left[1-\left(\frac{R \mathrm{~d} \Theta}{c \mathrm{~d} t}\right)^{2}\right] \mathrm{d} t
$$

we obtain the following Lagrange equation $(\dot{\Theta}=\mathrm{d} \Theta / \mathrm{d} t)$ : 


$$
\frac{\mathrm{d}}{\mathrm{d} t}\left\{\frac{\partial}{\partial \dot{\Theta}}\left[1-\left(\frac{R \dot{\Theta}}{c}\right)^{2}\right]\right\}=0
$$

from which:

$$
\frac{R^{2} \dot{\Theta}}{\left[1-\left(\frac{R \dot{\Theta}}{c}\right)^{2}\right]^{1 / 2}}=\text { constant }
$$

Now, the changes with time of the distance $R \Theta$ of the test-particle from point $\Theta=0$ are equal to $R \dot{\Theta}+\dot{R} \Theta$; but $\dot{R} \Theta$ is the speed of a point with a constant $\Theta$, which goes far from $\Theta=0$. Therefore the speed of the test-particle is $R \dot{\Theta}$, and its linear momentum $P$ is

$$
P=\frac{\varepsilon R \dot{\Theta}}{\left[1-\left(\frac{R \dot{\Theta}}{c}\right)^{2}\right]^{1 / 2}},
$$

if $\varepsilon$ is the very small mass of the particle.

Eq. (21) tells us that

$P R=$ constant .

It follows from this equation that $P \rightarrow \infty$, if $R \rightarrow 0$. And it follows from Friedmann's equations that

$$
\varrho R^{3}=\text { constant },
$$

from which we see that the mass density $\varrho$ of the galaxy "dust" goes to infinite if $R \rightarrow 0$. But with a very great mass density the momentum $P$ of the test-particle must be very small - as it is physically clear -, and not very great as it would follow from eqs. (23), (24). In conclusion, Friedmann's models of universe lose their physical meaning when $R \rightarrow 0$.

\section{APPENDIX}

As it is known, the perfect equivalence between the equations of motion of the Newtonian and Friedmannian models holds also with the addiction of the cosmological $\Lambda$-term.

Our formulation too allows the addiction of the $\Lambda$-term. Indeed, we can start from the following $\mathrm{d} s^{2}$, which is due to Weyl (1919) and Trefftz (1922) [12]: 


$$
\mathrm{d} s^{2}=\left(1-\frac{\alpha}{\mathcal{R}}-\frac{1}{3} \Delta \mathcal{R}^{2}\right) c^{2} \mathrm{~d} t^{2}-\left(1-\frac{\alpha}{\mathcal{R}}-\frac{1}{3} \Delta \mathcal{R}^{2}\right)^{-1} \mathrm{~d} \mathcal{R}^{2}-\mathcal{R}^{2}\left(\mathrm{~d} \vartheta^{2}+\sin ^{2} \vartheta \mathrm{d} \varphi^{2}\right)
$$

and then proceed as in sect. 1 .

Remark that if $\alpha=0$, eq. (A1) gives the $\mathrm{d} s^{2}$ of De Sitter's model (1917). -

LEGENDA concerning the following diagrams; putting $x:=\mathcal{R} / \alpha$, and $y:=[\mathrm{d} \mathcal{R} /(\mathrm{d} c t)]^{2}$, (eq. 2$)$ can be rewritten as follows:

$$
y(x)=\left(\frac{x-1}{x}\right)^{2}\left(1-|A| \frac{x-1}{x}\right)
$$

The six figures give diagrams of $y(x)$ for the following values of $|A|: 2 / 3$;

$0.5 ; 10^{-1} ; 10^{-3} ; 10^{-6} ; 0$. (Fig. 6 gives $y(x)$ for light-rays, $A=0$ ).

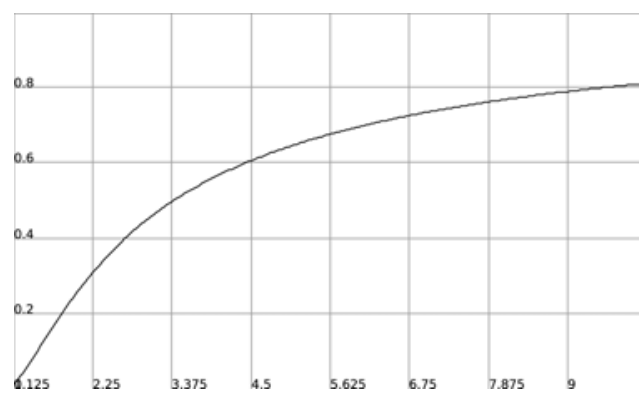

Fig. 1 - Diagram of $y(x)=[(x-1) / x]^{2}[1-(2 / 3) *(x-1) / x]$ for some values of $x$;

$(9 / 8) \leq x<+\infty ; \max (+\infty, 1 / 3) ;[y(9 / 8)]^{1 / 2}=0.106917$.

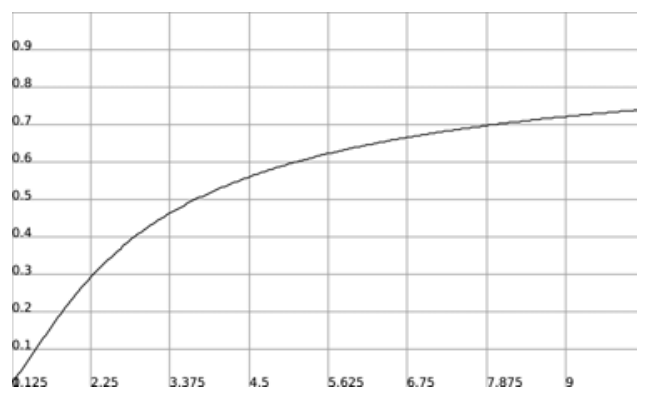

Fig. 2 - Diagram of $y(x)=[(x-1) / x]^{2}[1-0.5 *(x-1) / x]$ for some values of $x$; $(9 / 8) \leq x<+\infty ; \max (+\infty ; 0.5) ;[y(9 / 8)]^{1 / 2}=0.107981$. 


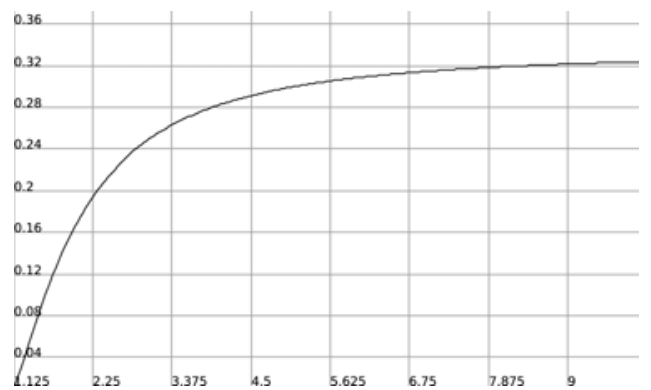

Fig. 3 - Diagram of $y(x)=[(x-1) / x]^{2}\left[1-10^{-1} *(x-1) / x\right]$ for some values of $x$; $(9 / 8) \leq x<+\infty ; \max (+\infty ; 0.9) ;[y(9 / 8)]^{1 / 2}=0.110492$.

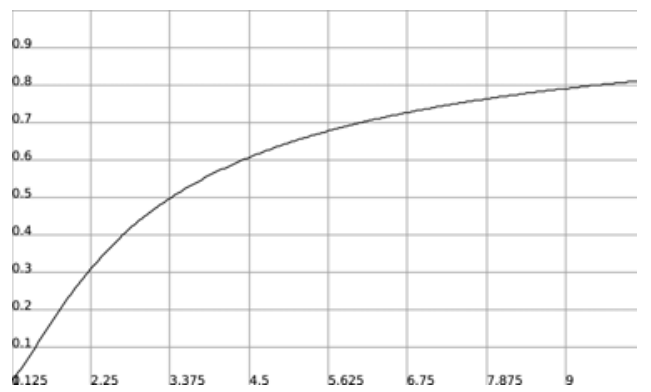

Fig. 4 - Diagram of $y(x)=[(x-1) / x]^{2}\left[1-10^{-3} *(x-1) / x\right]$ for some values of $x$; $(9 / 8) \leq x<+\infty ; \max \left(+\infty ; 1-10^{-3}\right) ;[y(9 / 8)]^{1 / 2}=0.111105$.

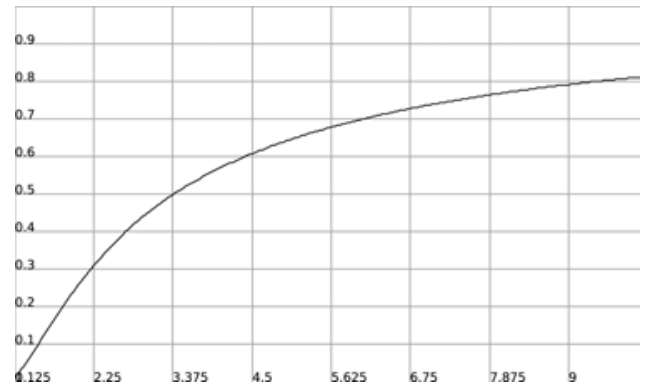

Fig. 5 - Diagram of $y(x)=[(x-1) / x]^{2}\left[1-10^{-6} *(x-1) / x\right]$ for some values of $x$; $(9 / 8) \leq x<+\infty ; \max \left(+\infty ; 1-10^{-6}\right) ;[y(9 / 8)]^{1 / 2}=0.111111$. 


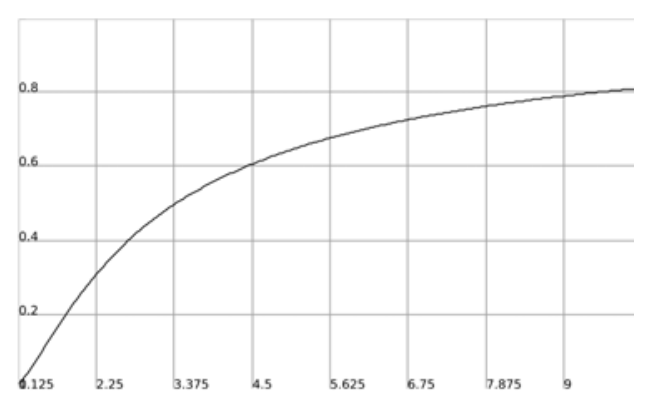

Fig. 6 - Diagram of $y(x)=[(x-1) / x]^{2}$ for some values of $x$;

$(9 / 8) \leq x<+\infty ; \max (+\infty ; 1.0) ;[y(9 / 8)]^{1 / 2}=1 / 9$.

\section{REFERENCES}

[1] E.A. Milne, Quart. J. Math, 5 (1934) 64; W.H. McCrea and E.A. Milne, ibid., 5 (1934) 73; W.H. McCrea, A.J., 60 (1955) 271.

[2] A. Friedmann, Z. Phys., 10 (1922) 377; A. Einstein, The Meaning of Relativity, Fifth Edition (Princeton University Press, Princeton, N.J.) 1955, App. for the Second Edition; M. v. Laue, Die allgemeiune Relativitätstheorie, 4. neubearbeitete Auflage (Friedr. Vieweg und Sohn, Braunschweig) 1956, sect. 51; G.C. McVittie, General Relativity and Cosmology (The University of illinois Press, Orbana) 1965, Chapt. 8. - And references therein.

[3] A. Loinger, arXiv:physics/0504018 (3 Apr. 2005).

[4] H. Karttunen et alii, Fundamental Astronomy, Fourth Edition (Springer-Verlag, Berlin, etc.) 2003, p. 386.

[5] D. Hilbert, Math. Annalen, 92 (1924) 1; also in Ges. Abh., Dritter Band (J. Springer, Berlin) 1935, p. 258.

[6] See, e.g., T. Levi-Civita, The Absolute Differential Calculus (Calculus of Tensors) - (Dover Pubblications, Inc., Mineola, N.Y.) 2005, Chapt. XII, sects. 11, 12, 13. - (First published by Blackie and Son Lim. - London and Glasgow, 1926.)

[7] See, e.g., L. Guzzo et alii, Nature, 451 (2006) doi710.1038/06555 ; L.J.M. Davies et alii, arXiv:1306.2634 v2 [astro-ph-CO] 4 Jul 2013; D. Burd et alii, arXiv:1309.5380 v1 [astro-ph-CO] 20 Sep 2013; L. Amendola et alii, Living Revs. - Relativity, 16 (2013) > Irr 20/3-6.

[8] See M. v. Laue, Die spezielle Relativitätstheorie, 6., neubearbeitete Auflage (Friedr. Vieweg u. Sohn, Braunschweig) 1955, sect. 1; and references therein.

[9] M. v. Laue [2].

[10] G.C. McVittie [2].

[11] H. Hönl, Ann. Physik, 6(1949) 169; M. v. Laue [2].

[12] M. v. Laue, book quoted in [2], sect. 43; and references therein. 